\title{
Simple Model for Respiratory Diseases
}

\author{
Puntani Pongsumpun* \\ Department of Mathematics, Faculty of Science, King Mongkut's Institute of Technology Ladkrabang, \\ Thailand. \\ * Corresponding author. Email: kppuntan@kmitl.ac.th \\ Manuscript submitted November 13, 2016; accepted February 2, 2017. \\ doi: 10.17706/ijbbb.2017.7.2.118-124
}

\begin{abstract}
Respiratory diseases can easily transmit between people. In this study, we formulate the simple model S-I-R-I (Susceptible-Infectious-Recovered-Infectious) for describing the transmission of this disease. The model is analyzed by using dynamical modeling method. After that, we simulate our equations by using numerical method. The numerical solutions are found to confirm our analytical results. Analysis of parameters are given.
\end{abstract}

Key words: Dynamical modeling method, model, numerical method, respiratory diseases, simulation.

\section{Introduction}

Respiratory diseases are caused by several types of viruses such as influenza virus, Para influenza virus, Respiratory syncytial virus etc. Almost cases of respiratory diseases caused by influenza virus [1]. Influenza virus has two types; types A and types B. The emerging infectious disease incidence has increased every year. These diseases are occurred via the respiratory tract. The spread of the disease is rapid and extensive. Emerging diseases have occurred since 1980, as the bird flu in Asia. The outbreak of severe acute respiratory infections (SARS) disease, Ebola, Middle East respiratory syndrome (MERS) caused serious effects on the health system. Most respiratory diseases, ie. SARS and Ebola, there is no vaccine. These diseases has spread rapidly and may infect again. Respiratory Disease Caused by an infection of the respiratory tract. Avian influenza virus has many subtypes but there are only some strains can infect human such as H5N1, H7N3, H7N7, H7N9, and H9N2 [2]. There was one elderly woman from Jiangxi Province, China, died of pneumonia in December 2013 from the H10N8 strain, the first human was confirmed to be died by that strain [3]. Most avian flu cases are a result of either handling dead infected birds or from contact with infected fluids. It can also be transmitted through contaminated surfaces and droppings. Most birds have only a mild form of the H5N1 strain, once domesticated birds such as chickens or turkeys are infected, it can die because the birds are often in closed contact. Because of low hygiene conditions, there is a large threat of this disease in Asia. This disease can be easily transmitted from birds, human-to-human transmission is more difficult without prolonged contact [4]. Some strains of avian influenza are present in the intestinal tract of large numbers of shore birds and water birds, but these strains are rarely caused infection [5]. SARS is a viral respiratory disease of zoonotic origin caused by the SARS coronavirus (SARS-CoV). The outbreak is occurred between November 2002 and July 2003 in Southern China. There were about 8,096 cases and 774 deaths reported from 37 countries [6]. The most cases from Hong Kong [6] (9.6\% fatalityrate) according to the World Health Organization (WHO) [7]. There is no cases of SARS have been reported worldwide since 2004 [8]. In 1968, Rvachev [9] developed the spatial-temporal model by 
using SIR model to study the connection of SIR model with the real epidemic situation in Soviet Union. After that, Rvachev et al. extended their model by finding the effect of air travel with the transmission of this disease [10]. In 1988, Flahault et al. [11] studied and formulated the mathematical model describing the transmission of Influenza in France. After that [12], they studied and constructed mathematical model describing the transmission of influenza in Europe. In 2014, Chowell et al. [13], studied the transmission model of Mers by considering the data of Mers during April and October 2013, the basic reproduction number and disease severity.

In this study, we analyze the simple model for describing the transmission of respiratory diseases and numerical results are found to show the behaviors of solutions.

\section{Transmission Model}

Mathematical model is applied to describe the transmission of the diseases are occurred long time ago. To model the transmission of the disease, we have to know process about the disease and what are its transmission cycles. Respiratory disease can transmit between humanthrough contaminated surfaces and droppings. To construct the mathematical model, we need to define variables and parameters for describing the transmission of this disease. We define the variables as follows:

$S(t)$ is the number of susceptible human,

$I(t)$ is the number of infectious human,

$R(t)$ is the number of recovered human.

The diagram can be written as

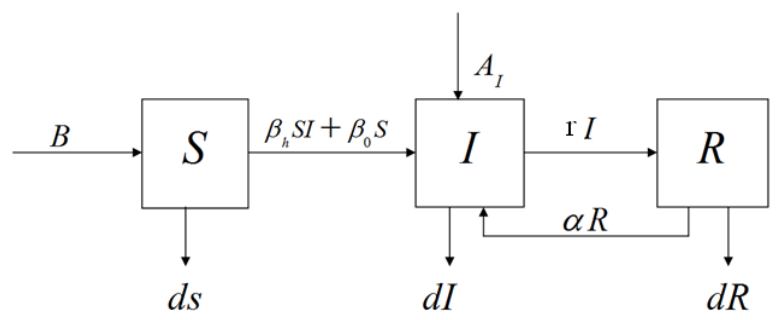

Fig. 1. Diagram of our model equations.

From the above diagram, we can have following equations.

$$
\begin{aligned}
& S^{\prime}(t)=B-\left(\beta_{h} S(t) I(t)+\beta_{o} S(t)\right)-d S(t) \\
& I^{\prime}(t)=\left(\beta_{h} S(t) I(t)+\beta_{o} S(t)\right)+\alpha R(t)+A_{I}-(r+d) I(t) \\
& R^{\prime}(t)=r I(t)-(\alpha+d) R(t)
\end{aligned}
$$

where $B$ is the number of human population at the beginning time,

$\beta_{h}$ is the infection rate of respirator y diseases between human,

$\beta_{o}$ is the infection rate of respirator y diseases from the other factors,

$r$ is the recovery rate of cases,

$\alpha$ is the rate at which recovered human become to be infectious human again,

$A_{i}$ is the initial number of cases,

$d$ is the natural death rate of human. 
$N$ is the total human.

\subsection{Analytical Results}

The steady states can be found from setting (1) to zero, then the steady states are

1) The first steady state $\left(S_{1}^{*}, I_{1}^{*}, R_{1}^{*}\right)$

where

$$
\begin{gathered}
S_{1}^{*}=\frac{B}{\left(\beta_{0}+d+\beta_{h} I_{1}^{*}\right)} \\
R_{1}^{*}=\frac{I_{1}^{*} r}{(a+d)},
\end{gathered}
$$

and $I_{1}^{*}$ is given by

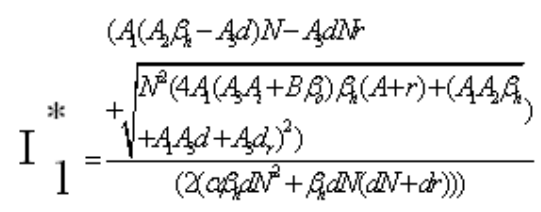

where $A_{1}=a+d, A_{2}=A_{i}+B, A_{3}=B_{0}+d$

2) The second steady state $\left(S_{2}^{*}, I_{2}^{*}, R_{2}^{*}\right)$ where

$$
\begin{gathered}
S_{2}^{*}=\frac{B}{\left(\beta_{0}+d+\beta_{h} I_{2}^{*}\right)}, \\
R_{2}^{*}=\frac{I_{2}^{*} r}{(a+d)}
\end{gathered}
$$

and $I_{2}^{*}$ is defined by

$$
\begin{aligned}
& \left(A\left(A_{2} \beta_{2}-A d\right) N-A d N N^{5}\right.
\end{aligned}
$$

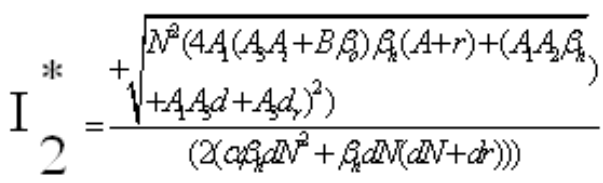

We analyze the stability of the steady states by looking the sign of all eigenvalues. For the first steady state, we found that all eigenvalues have negative signs when $R_{0}<1$.

For $R_{0}>1$, all eigenvalues of the second steady state have negative real parts. $R_{0}$ is defined as

$$
R_{0}=\frac{4 A_{1}\left(A_{3} A_{i}+B \beta_{0}\right) \beta_{h} d\left(A_{1}+r\right)+\left(-A_{1} A_{2} \beta_{h}+A_{1} A_{3} d+A_{3} d r\right)^{2}}{\left(-A_{1} A_{2} \beta_{h}+A_{1} A_{3} d+A_{3} d_{r}\right)^{2}} .
$$


If all eigenvalues of the steady states have negative real parts, then that steady state will be local stable [14].

\subsection{Numerical Results}

We simulate our equations by using numerical method. We consider the behaviors of solutions for the different set of parameters as follows:

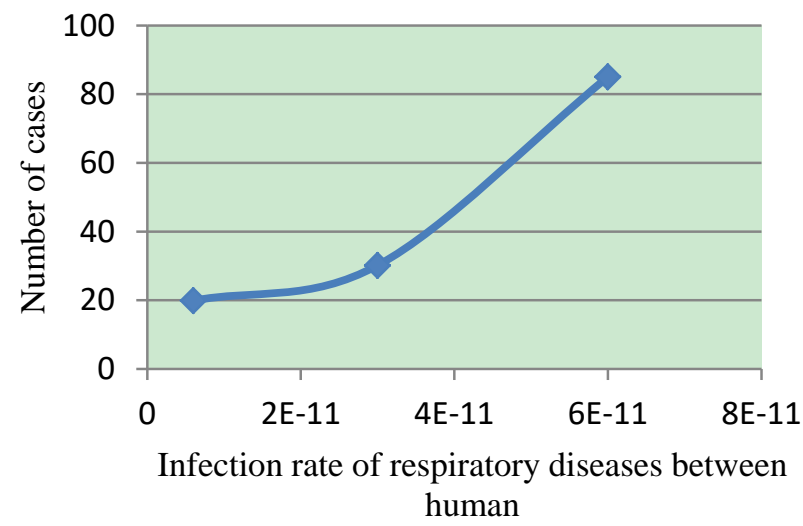

Fig. 2. Number of cases for the different Infection rates of respiratory diseases between human.

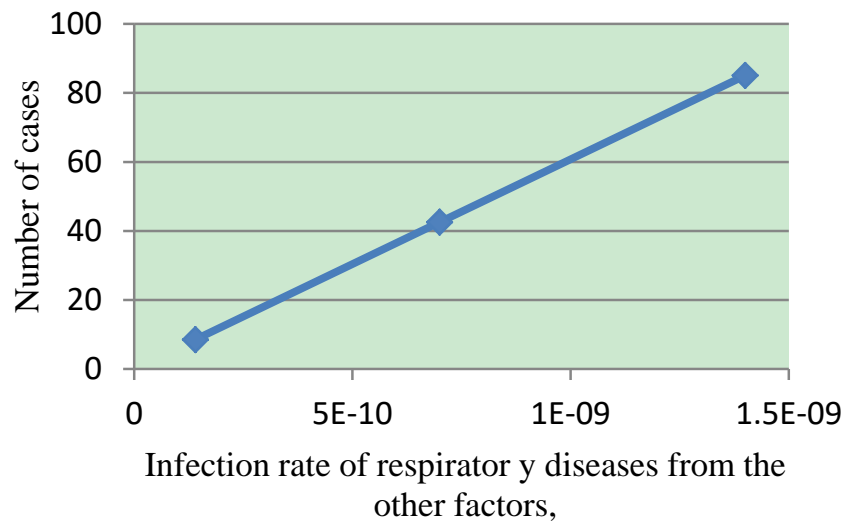

Fig. 3. Number of cases for the different infection rate of respirator y diseases from the other factors.

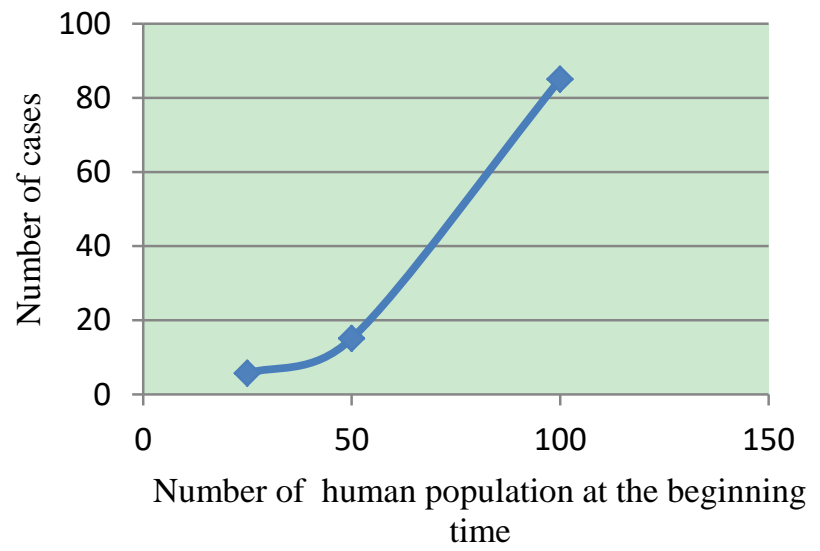

Fig. 4. Number of cases for the different number of human population at the beginning time?. 


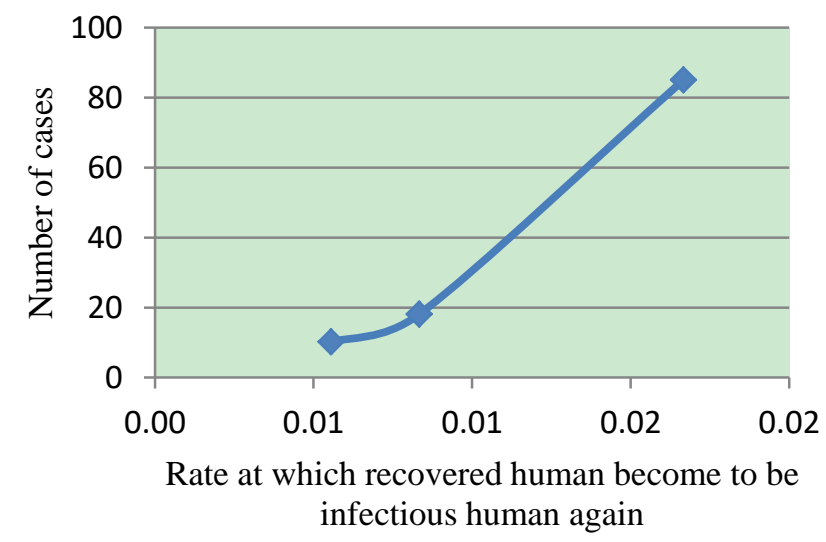

Fig. 5. Number of cases for the different rate at which recovered human become to be infectious human again.

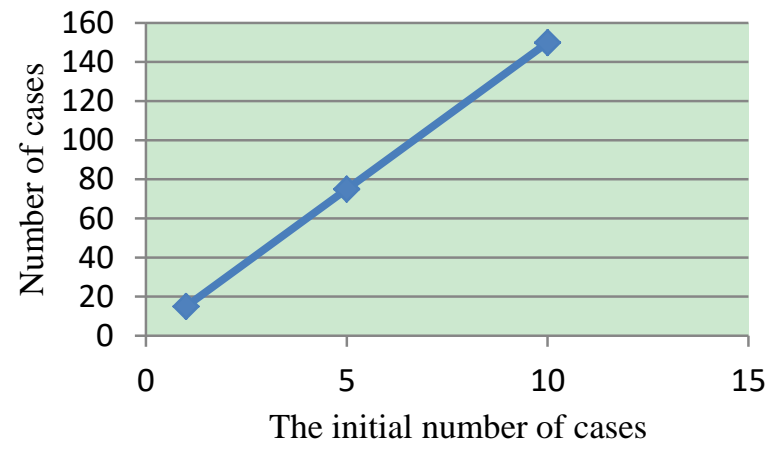

Fig. 6. Number of cases for the different initial number of cases.

Table 1. Parameters of Our Model

\begin{tabular}{|c|c|c|c|}
\hline Parameters & \multicolumn{3}{|c|}{ Values } \\
\hline$\beta_{h}$ & 0.00000000006 & 0.00000000003 & 0.000000000006 \\
\hline$\beta_{o}$ & 0.0000000014 & 0.0000000007 & 0.00000000014 \\
\hline $\mathrm{B}$ & 100 & 50 & 25 \\
\hline $\mathrm{a}$ & 0.02 & 0.01 & 0.01 \\
\hline$\beta_{h}$ & 1 & 5 & 10 \\
\hline
\end{tabular}

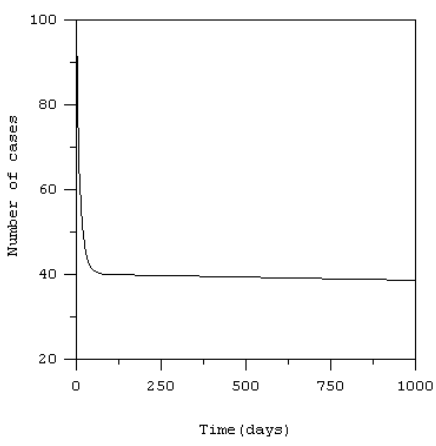

a)

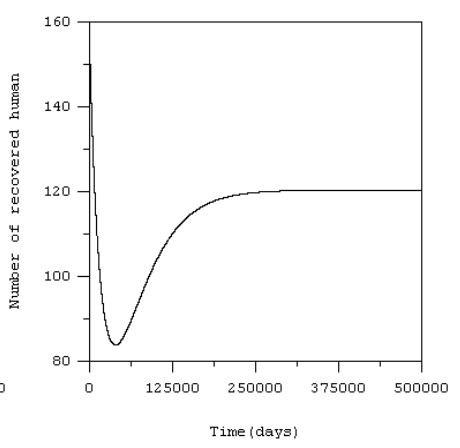

b)

Fig. 7. Times series solution behaviors of our model. The parameters are $\mathrm{B}=100, \beta_{o}=0.0000000014, \mathrm{~d}=$ 1/(365*70), $\beta_{h}=0.00000000003, \alpha=1 / 60 ; A_{i}:=0 ; \mathrm{r}:=1 / 15 . R_{0}=1.1$. a) For respiratory disease cases.

b) For the recovered human. 


\section{Conclusion}

In this paper, we study the mathematical models for describing the transmission of respiratory disease. S-I-R-I is used for this study. We analyzed our model by dynamical modeling method. The stability condition is used to be guided for reducing the transmission of this disease. The local stability condition of two steady states is determined by $R_{0}$. If $R_{0}<1$, then the first steady will be stable. The second steady state will be stable for $R_{0}>1$. The following figures are the example of numerical results for $R_{0}>1$.

From Fig. 7, we can see that when we use the above parameters, the cases can be reduced and also the recovered human increased until they converge to the second steady state.

We found that the parameters $\beta_{h}, \beta_{o}, \mathrm{~B}$, a and $A_{i}$ are influenced to the number of cases. From Fig. 2 to Fig. 6, we can see that if the infection rate of respiratory diseases between human, the infection rate of respiratory diseases from the other factors, the number of human population at the beginning time, the rate at which recovered human become to be infectious human again and the initial number of cases are higher, then the number of cases are higher too.

\section{Acknowledgment}

The author would like to thank King Mongkut's Institute of Technology Ladkrabang and National Research Council of Thailand for financial support.

\section{References}

[1] Viruses. Retrieved from the website: http://www.cdc.gov/flu/about/viruses/

[2] Silva, M. E., Cherry, J. D., Wilton, R. J., Ghafouri, N. M., Bruckner, D. A., \& Miller, M. J. (August 1999). Acute fever and petechial rash associated with influenza a virus infection. Clinical Infectious Diseases, 29(2), 453-454. doi:10.1086/520240.PMID 10476766.

[3] China reports first human H10N8 avian flu death - CNN.com. (2013). Retrieved from the website: http://www.cidrap.umn.edu/news-perspective/2013/12/china-reports-first-human-case-h10n8-avia n-flu

[4] Influenza (Seasonal) Fact sheet $\mathrm{N}^{\circ} 211$. (2014). Retrieved from the website: http://www.who.int/mediacentre/factsheets/fs211/en/

[5] Recommendations for Worker Protection and Use of Personal Protective Equipment (PPE) to Reduce Exposure to Highly Pathogenic Avian Influenza A H5 Viruses: Avian Influenza (Flu). Retrieved from the website: www.cdc.gov. Retrieved 2015-07-25

[6] Smith, R. D. (2006). Responding to global infectious disease outbreaks, Lessons from SARS on the role of risk perception, communication and management. Social Science and Medicine, 63(12), 3113-3123.

[7] WHO. Summary of probable SARS cases with onset of illness from 1 November 2002 to 31 July 2003. Retrieved from the website: http://www.who.int/csr/sars/country/table2004_04_21/en/

[8] SARS (severe acute respiratory syndrome). NHS Choices. United Kingdom: National Health Service. 2014-10-03. Retrieved from the website: http://www.nhs.uk/conditions/sars/pages/introduction.aspx

[9] Rvachev, L. A. Modeling experiment of a large-scale epidemic by means of a computer. Transactions of USSR Academy of Sciences Series: Trans Mathematics and Physics, 1668(180), 294-296.

[10] Rvachev, L. A., \& Longini, I. M. (1985). A mathematical model for the global spread of influenza. Mathematical Biosciences, 75, 3-22.

[11] Flahault, A., et al. (1988). Modelling the 1985 influenza epidemic in France. Statistics in Medicine, 7, 1147-1155. 
[12] Flahault, A., et al. (1994). A mathematical model for the European spread of influenza. European Journal of Epidemiology, 10, 471-474.

[13] Gerardo, C., et al. (2014). Syntheszing data and models for the spread of MERS - CoV, 2013: Key role of the index cases and hospital transmission.Epidemics, 9, 40-51.

[14] Leah, E.K. (1988). Mathematical models in biology. Random.

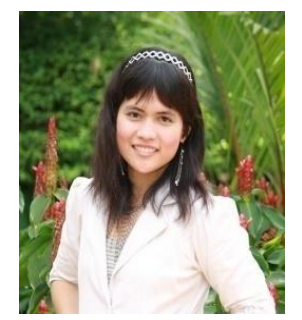

P. Pongsumpun received her bachelor of science in mathematics (second class honors) and the doctor of philosophy in mathematics (international programme) from Mahidol University, Thailand. From 2004 to 2012, she was an as assistant professor of Mathematics. From 2012 till date, she is an associate professor of mathematics, thesis Ph.D. and M.Sc. advisors in King Mongkut's Institute of Technology Ladkrabang, Thailand. equations and numerical analysis. 